\title{
A randomised phase IIb trial to assess the efficacy of ReCharge ice cream in preventing chemotherapy-induced diarrhoea
}

\author{
D. Perez ${ }^{1,9}$ - K. J. Sharples ${ }^{2,3} \cdot$ R. Broom ${ }^{4}$ - M. Jeffery ${ }^{5}$ - J. Proctor $^{3} \cdot$ V. Hinder ${ }^{3}$.

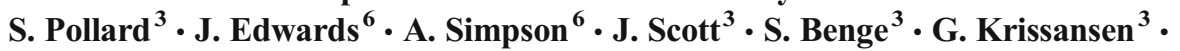

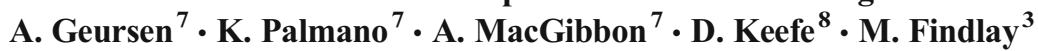

Received: 13 September 2014 / Accepted: 27 April 2015 /Published online: 28 May 2015

(C) Springer-Verlag Berlin Heidelberg 2015

\begin{abstract}
Purpose Chemotherapy-induced diarrhoea (CID) has a significant impact. A medicinal food product (ReCharge) containing iron-saturated lactoferrin and anhydrous milk fat reduces the detrimental effects of chemotherapy on the gut in animals. We report results of a randomised blinded placebocontrolled phase IIb trial investigating the efficacy and safety of ReCharge in preventing CID.

Methods Eligible patients were adults due to start the first cycle of a 2- or 3-week-cycle chemotherapy regimen, had an Eastern Cooperative Oncology Group (ECOG) status of 3 or less, had adequate haematological, liver and renal function and provided written informed consent. Patients (197) were randomised to ReCharge or placebo. They
\end{abstract}

\section{Perez}

david.perez@southerndhb.govt.nz

1 University of Otago Medical School, Dunedin, New Zealand

2 Department of Preventive and Social Medicine, Dunedin School of Medicine, University of Otago, Dunedin, New Zealand

3 Cancer Trials New Zealand, University of Auckland Faculty of Medical \& Health Sciences, Auckland, New Zealand

4 Auckland Regional Cancer \& Blood Service, Auckland District Health Board, Auckland, New Zealand

5 Medical Oncology, Canterbury District Health Board, Christchurch, New Zealand

6 Wellington Blood \& Cancer Centre, Capital \& Coast District Health Board, Kilbirnie, New Zealand

7 LactoPharma NZ LTD - Fonterra Co-operative Group Ltd., Hamilton, New Zealand

8 University of Adelaide, Adelaide, Australia

9 Department of Oncology, Dunedin Hospital, Private Bag 1921, Dunedin 9054, New Zealand consumed 100-g study product for 2 weeks before and 6 weeks after starting chemotherapy, completed daily diaries for 8 weeks and attended clinic visits until 12 weeks (2-week cycles) or 14 weeks (3-week cycles). The primary outcome was days with CID.

Results The mean number of days with diary-recorded CID was marginally but not statistically significantly lower on ReCharge than placebo $(-2.0,95 \% \mathrm{CI}(-4.7$ to 0.7$), p=0.2)$. The proportion reporting diarrhoea in the previous cycle at the clinic visit was $30 \%$ lower $(p=0.012)$ on ReCharge. Missing diary data may have contributed to the discrepancy. No significant differences were found in quality of life or other adverse events.

Conclusions We found no clear evidence that ReCharge reduced CID as measured by patient self-report diary. The converse finding of benefit as recorded at clinic visits and incomplete adherence to diary completion indicates that further research is required into methods for measuring CID.

Keywords Chemotherapy · Diarrhoea - Myelosuppression · Quality of life · Lactoferrin

\section{Introduction}

Chemotherapy-induced diarrhoea (CID) due to mucositis of the bowel has a significant impact on patients' quality of life and can impact on the ability to deliver effective treatment [1]. It is a common side effect of many chemotherapy regimens [2]. The reported incidence of diarrhoea (any grade) associated with the use of cytotoxic drugs ranges from $30 \%$ (docetaxel), $50 \%$ (5-fluorouracil and capecitabine), to $80 \%$ (irinotecan) [3]. These incidence figures underestimate the true impact of diarrhoea as patients frequently use anti- 
diarrhoeal agents to control their symptoms. Treatments for mucositis are palliative at best. [4].

ReCharge is a medicinal food product developed to ameliorate the gut damage caused by chemotherapy. It contains two milk-derived bioactive components, namely, anhydrous milk fat (AMF) and fully iron-saturated lactoferrin (natural lactoferrin is $15 \%$ saturated). Lactoferrin, a protein that occurs naturally in human and cow's milk as well as in other bodily secretions, has a role in the body's natural defence system. AMF is a commercial food product that has been widely used in the food industry for many years. Animal data have shown that both iron-saturated lactoferrin and milk fat prevent chemotherapy-induced gut damage and aid recovery from neutropenia [5-7] and that the two components work synergistically (unpublished results). There have been over 20 studies in humans investigating lactoferrin's effect on iron absorption [8], Helicobacter pylori eradication [9], hepatitis $\mathrm{C}$ eradication [10], a variety of other infections [11], colonic polyps [12] and modulation of tumour growth [13], including two short-term trials involving high doses of fully iron-saturated lactoferrin $[8,14]$. No safety concerns were identified by these trials.

This report describes a randomised, placebo-controlled, double-blinded, phase IIb trial investigating the efficacy and safety of ReCharge in preventing CID in patients undergoing chemotherapy. The primary objective was to assess the efficacy of ReCharge ice cream formulation in reducing days with CID.

\section{Methods}

Patients were recruited from seven New Zealand centres between August 2009 and May 2012. Patients 18 years or over with advanced or early stage (following a protocol amendment in December 2009) cancer of any type were eligible for the study if it had been more than 4 weeks since their last chemotherapy regimen; they were to start a first, second or third line regimen with cycles of 2 or 3 weeks and including at least one of capecitabine, docetaxel, paclitaxel, 5-FU or irinotecan in the regimen; had Eastern Cooperative Oncology Group (ECOG) performance status of 3 or better; had haemoglobin $>90 \mathrm{~g} / \mathrm{L}$, neutrophil count $>1.5 \times 10^{9} / \mathrm{L}$, platelets $>100 \times 10^{9} / \mathrm{L}$, bilirubin $<2 \times$ upper limit, AST/ALT/ALP $<2 \times$ upper limit, albumin $>30 \mathrm{~g} / \mathrm{L}$ and creatinine clearance $>50 \mathrm{~mL} / \mathrm{min}$; were able to tolerate ice cream; and provided written informed consent. Exclusion criteria included intolerance of cow's milk, celiac disease, presence of a stoma, treatment with oxaliplatin, use of iron supplements within 3 days of starting study treatment, haemochromatosis, type 1 or poorly controlled type 2 diabetes, planned G-CSF support, participation in another clinical study within 4 weeks of inclusion and concurrent abdominal radiation.
ReCharge was formulated as an ice cream with $16 \%$ anhydrous milk fat, $2.5 \%$ fully iron-saturated bovine lactoferrin, $7 \%$ skim milk powder and $14 \%$ sugar plus stabilisers and emulsifiers. An inert placebo ice cream was used (soybeanoil-based, matched for colour, taste and appearance) which contained the same constituents as ReCharge apart from absence of iron saturated lactoferrin and anhydrous milk fat, the latter being replaced with $16 \%$ soybean oil.

Eligible patients were randomised 1:1 to ReCharge or placebo ice cream. Products were manufactured in a facility accredited by the Ministry for Primary Industries with an approved Hazard Analysis and Critical Control Point plan. They were administered orally as one $100 \mathrm{~g}$ serving of ice cream daily for 10-18 days pre-chemotherapy (for priming the gut $[5,6])$ and continued for 6 weeks from the start of chemotherapy (a total of 8 weeks \pm 4 days). Randomisation was stratified and blocked by length of planned chemotherapy cycle ( 2 vs 3 weeks) and study site using random block sizes of 4 or 6 . If chemotherapy was discontinued, patients were encouraged to continue taking study product wherever possible.

Patients were assessed at clinic at baseline, the start of study product and the start of each chemotherapy cycle until the end of week 8. A haematology profile and biochemistry profile for renal and hepatic function were performed at baseline, and haematology counts were repeated in cycle 1 only on days $3,5,8,10,12,15,18$ and 21 . Thereafter, blood tests were done according to the institution's standard practice. Patients completed daily diaries from randomisation until the end of week 8. A final study visit was carried out at the end of 12 or 14 weeks for patients on 2- and 3-week cycles, respectively.

The primary outcome measure was the numbers of days with CID using data from the patient's daily diary. Following the Common Terminology Criteria for Adverse Events (CTCAE) Version 3.0, we defined any day with at least one bowel motion (BM) over the baseline as a day with CID. The baseline level was estimated before randomisation from patient recall of the average number of BM over a 3-day period.

The secondary outcome measures were incidence of CID (1 or more days with CID); diarrhoea-related quality of life (FACIT-D diarrhea subscale) measured at each study visit; maximum severity of diarrhoea (based on recall at the clinic visit and graded according to CTCAE version 3.0); duration and severity of neutropenia, measured as the area under the curve (AUC) during the first cycle of chemotherapy; and health-related QoL and safety (adverse events between the first dose of study product and 28 days after the last dose coded using CTCAE version 3.0). Compliance with study product was recorded in patient diaries. Compliance with chemotherapy was measured by calculating the proportion of the planned dose delivered from start of chemotherapy to end of study product. Use of anti-diarrhoeal medication was also recorded by patients in their diaries. The primary intention-totreat analysis compared the mean number of days with CID 
between 2 weeks post-randomisation (planned chemotherapy start date) and 8 week post-randomisation on ReCharge and placebo using a linear regression. An efficacy analysis compared the mean number of days with CID from 2 weeks after start of chemotherapy for 42 days. The relative reduction in rate of CID was calculated using a Poisson regression model with the number of days of diary data as exposure time and robust standard errors. Cumulative incidence of CID and the proportions with grade 1-4 or grade 3-4 diarrhoea were compared using relative risk regression with robust standard errors [15]. The overall proportions of grade 3 or higher AEs were compared using a chi-square test. Quality of life scores and neutrophil counts were compared using linear mixed models with REML estimates of variance components and assumed correlation structures of compound symmetry (QoL) and unstructured (neutrophils). Regression models were adjusted for the randomisation stratification factors (length of planned chemotherapy cycle and whether or not the regimen included capecitabine, irinotecan or 5-FU). No formal adjustments were made for multiplicity of secondary outcomes.

The study was designed with three decision regions for the estimated primary treatment effect [16]. Patient recall data from previous local trials gave a mean (SD) number of days with CID of 10 (12) [17]. Inflating to account for underreporting in the clinic, we assumed a mean of 12 days in the control arm and considered a reduction of 4 days (30\%) to be clinically meaningful. With a sample size of 100 per arm, the trial has the following operating characteristics:

Region 1 If the estimated treatment effect was a reduction of 2 days or fewer of CID, we would conclude that ReCharge is unlikely to be superior to placebo, with a $12.4 \%$ chance of a false negative conclusion.

Region 2 An estimated treatment effect between 2 and 3.3 days of CID would indicate sufficient promise to warrant further investigation in a larger trial.

Region 3 An estimated treatment effect of 3.3 days would indicate that the mean number of days with CID was lower on ReCharge than placebo, with a $2.5 \%$ chance of a false positive conclusion.

Ethical approval was given by the Multi-Region Ethics Committee (MEC 09/03/031), and regulatory approval by the Standing Committee on Therapeutic Trials (TT50-8315). The trial was monitored by an independent Data Monitoring Committee.

\section{Results}

Of the 1241 patients screened, 197 were randomised (Fig. 1). Of the 197 patients randomised, 43 (22\%) did not meet all eligibility criteria, 23 on ReCharge and 20 on placebo. Eighteen were given exemptions before randomisation; 13 of these were just below cutoffs for renal function, liver function and/ or haematological profile, for 1 AST was not done, 3 would have study product for less than 10 days before planned chemotherapy, and 1 had more than 2 previous lines of chemotherapy. One patient on placebo was found to have a colostomy and was withdrawn from the trial. Of the remainder without exemptions, $16 \mathrm{did}$ not have one of the required blood tests before randomisation ( 9 were missing AST, caused by a change in laboratory practice), 3 were below cutoffs for renal function or platelets, 2 had less than 28 days since their last chemotherapy cycle, 2 had more than two previous lines of chemotherapy, and for 1 ECOG performance status was not recorded. The Steering Committee decided it was safe for these patients to continue on study. Baseline characteristics are shown in Table 1.

Three patients died (one on ReCharge and two on placebo) and 14 withdrew from the study before 8-week follow-up (chemo period) was complete (8 on ReCharge, 6 on placebo) (Fig. 1). Twelve patients did not provide any diary information after the 2-week pre-chemotherapy period (seven on ReCharge, five on placebo). The numbers (proportions) of patients providing any data for the primary analysis were $89 / 102$ (87\%) on ReCharge and 84/95 (88\%) on placebo, and the proportions providing at least $80 \%$ complete data were $71 \%$ on ReCharge and $72 \%$ on placebo.

The primary intention-to-treat analysis found that the mean number of days with CID was lower on ReCharge than on placebo (difference in means $-2.0,95 \%$ confidence interval $(-4.7$ to 0.7$))$, but the difference was not statistically significant $(p=0.2)$ ) (Table 2). Adjustment for baseline imbalances including age, sex, pre-existing bowel conditions, cancer site and disease status reduced the size of the estimated difference to -1.6 (95\% CI ( -4.3 to 1.1$), p=0.2)$. This result falls in region 1 of the three-region design, indicating that ReCharge is unlikely to be superior to placebo. Secondary analyses compared the relative reduction in rate of CID and the overall proportion reporting any CID, but neither difference was statistically significant ( $p=0.1$ and $p=0.06$ respectively, Table 2 ). However, there was a $30 \%$ reduction in patients reporting grade 1 or higher diarrhoea adverse events in the clinic ( $p=$ $0.02)$. This latter analysis includes all patients who continued to attend clinic visits, so it may be a more complete and reliable estimate of the effect of treatment on CID. However, it was not the pre-specified primary endpoint so it should be interpreted with caution due to multiplicity of secondary outcomes. The slightly higher rate of use of anti-diarrhoeal agents by ReCharge patients may also have affected the comparisons of CID (Table 2). Of the 197 patients, 66 recorded use of antidiarrhoeal medication in their daily diary. Of these, seven were classified as not having CID according to the primary outcome (daily diary) measure (four on ReCharge and three on 


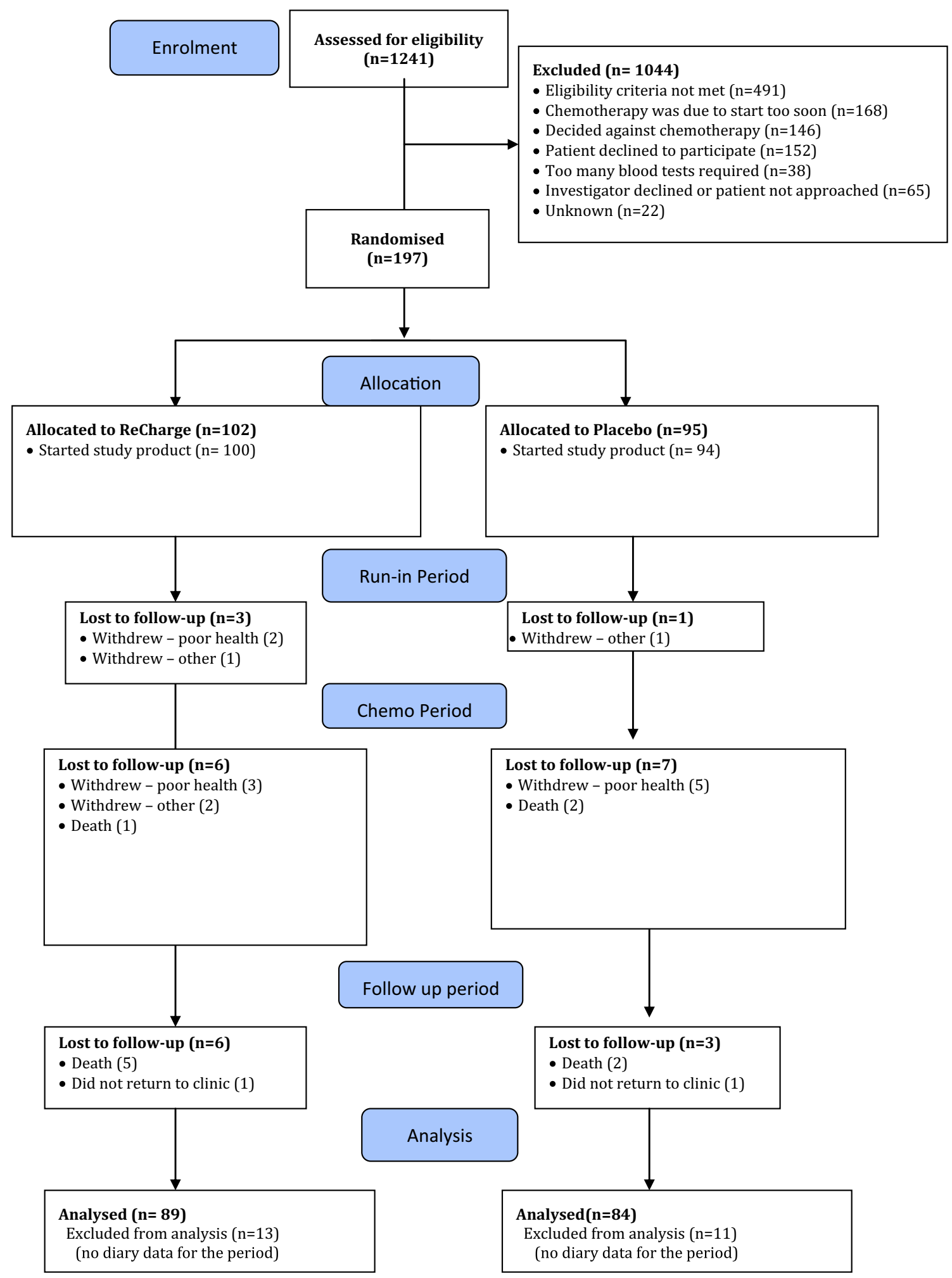

Fig. 1 Disposition of patients from screening to the end of the study

placebo), so the potential for bias in the diary measure due to use of anti-diarrhoeal medication was low. However, for the secondary outcome measure based on clinic CTCAE grades, of 37 patients on ReCharge who used anti-diarrhoeal medications, 17 were not classified as having CTCAE graded diarrhoea, whereas only 3 of 29 on placebo did not have CTCAE graded diarrhoea, so it is possible that there is bias in this clinic measure. 
Table 1 Baseline characteristics of ReCharge and placebo groups

\begin{tabular}{|c|c|c|c|c|}
\hline & \multicolumn{2}{|c|}{ ReCharge } & \multicolumn{2}{|c|}{ Placebo } \\
\hline & \multicolumn{2}{|c|}{$(N=102)$} & \multicolumn{2}{|c|}{$(N=95)$} \\
\hline & $n$ & $(\%)$ & $n$ & $(\%)$ \\
\hline Age (median, IQ range) & 59.2 & (47.7 to 68.0$)$ & 61.3 & (50.0 to 71.8$)$ \\
\hline Sex (female) & 65 & $(63.7)$ & 64 & $(67.4)$ \\
\hline Bowel obstruction within last 4 weeks & 4 & $(3.9)$ & 2 & $(2.1)$ \\
\hline Other pre-existing bowel condition ${ }^{a}$ & 10 & $(9.8)$ & 5 & $(5.3)$ \\
\hline Primary cancer site: GI & 41 & $(40.2)$ & 34 & $(35.8)$ \\
\hline Primary cancer site: breast & 44 & $(43.1)$ & 48 & $(50.5)$ \\
\hline Primary cancer site: other ${ }^{\mathrm{b}}$ & 17 & $(16.7)$ & 13 & $(13.7)$ \\
\hline Prior chemotherapy 1 cycle & 27 & $(26.5)$ & 22 & $(23.2)$ \\
\hline Prior chemotherapy 2 cycles & 6 & $(5.9)$ & 9 & $(9.5)$ \\
\hline Prior chemotherapy 3 cycles & 4 & $(3.9)$ & 4 & $(4.2)$ \\
\hline ECOG 0 & 60 & $(58.8)$ & 56 & $(58.9)$ \\
\hline 1 & 37 & $(36.3)$ & 35 & $(36.8)$ \\
\hline 2 and 3 & 5 & $(4.9)$ & 4 & $(4.3)$ \\
\hline \multicolumn{5}{|l|}{ Average bowel count per day } \\
\hline Median (IQ range) & 1.5 & $(1.0$ to 2.0$)$ & 1.0 & $(1.0$ to 2.0$)$ \\
\hline Unknown & 2 & & 3 & \\
\hline \multicolumn{5}{|l|}{ Planned chemotherapy includes: } \\
\hline 5-FU/capecitabine & 70 & $(68.6)$ & 69 & $(72.6)$ \\
\hline Irinotecan & 19 & $(18.6)$ & 19 & $(20.0)$ \\
\hline Taxane & 29 & $(28.4)$ & 21 & $(22.1)$ \\
\hline \multicolumn{5}{|l|}{ Length of planned chemotherapy cycle } \\
\hline 2 weeks & 16 & $(15.7)$ & 12 & $(12.6)$ \\
\hline 3 weeks & 86 & $(84.3)$ & 83 & $(87.4)$ \\
\hline \multicolumn{5}{|l|}{ Neutrophils $\left(10^{9} / \mathrm{L}\right)$} \\
\hline Median (IQ range) & 4.6 & (3.3 to 5.9 ) & 4.6 & (3.2 to 5.9$)$ \\
\hline
\end{tabular}

${ }^{\text {a }}$ Constipation, irritable bowel, flatulence, gastritis

${ }^{\mathrm{b}}$ Lung, prostate, ovarian, head and neck, uterus, unknown primary
The first efficacy analysis used data from the start of chemotherapy for 42 days, which excluded patients (nine on ReCharge, two on placebo) who did not start chemotherapy. The adjusted difference in mean number of days with CID was $-2.46(95 \% \mathrm{CI}-5.1$ to $0.18, p=0.07)$ and the adjusted rate ratio $0.76(0.57$ to $1.01(p=0.06)$. The majority of patients who stopped chemotherapy also stopped providing diary data, so further restriction to those completing chemotherapy, and to those who stayed on study product while on chemotherapy had little effect on the estimate of efficacy.

The difference in mean FACIT-D scale scores is given in Table 3. There were no statistically significant differences between the treatment arms. The confidence intervals excluded differences of more than 1.5 on the diarrhoea subscale, and more than 2 across all other subscales. Overall $85 \%$ of the QoL questionnaire forms were returned. For patients on regimens not including capecitabine, neutrophil counts rose in the first 3 days after start of chemotherapy then dropped (Fig. 2), but for those on capecitabine, there was no spike. For patients on 3-week cycles, the difference in area under the curve (AUC) (ReCharge-placebo) was -3.51 (95\% CI -23.2 to 15.8$) p=0.7$, and for patients on 2-week cycles, 0.17 (95\% CI ( -12.5 to 12.8$), p=0.97$. There were 3 patients who experienced grade 3 febrile neutropenia on each arm, and $12(12 \%)$ patients on ReCharge and $18(19 \%)$ on placebo experienced grade 3 or higher neutropenia.

Eight patients died from their cancer during the trial, five on ReCharge and three on placebo. There was one noncancer death on ReCharge (due to GI necrosis) and one on placebo (cardiac ischemia/infarction). There was no statistically significant difference in overall number of adverse events grade 3 or higher ( $p=0.1$, Table 4$)$. Furthermore, there were no significant differences in adverse event rates in any of the haematological or gastrointestinal subcategories apart from the differences in diarrhoea mentioned above. 
Table 2 Comparison of diarrhoea outcomes in ReCharge and placebo groups (intention-totreat analysis)

\begin{tabular}{|c|c|c|c|c|}
\hline \multirow[b]{2}{*}{ Number with any diary data } & \multicolumn{2}{|c|}{ ReCharge $N=102$} & \multicolumn{2}{|c|}{ Placebo $N=95$} \\
\hline & 89 & $(87.3)$ & 84 & $(88.4)$ \\
\hline \multicolumn{5}{|l|}{ Number of days of CID (diary data) } \\
\hline Median (IQ range) & 6.0 & (1.0 to 13.0$)$ & 6.5 & (2.0 to 15.5$)$ \\
\hline Mean (SD) & 8.3 & $(8.4)$ & 10.0 & $(9.5)$ \\
\hline Adjusted $^{\mathrm{a}}$ difference in means $(95 \% \mathrm{CI})$ & -2.0 & $(-4.7$ to 0.7$)$ & $p=0.2$ & \\
\hline Adjusted $^{\mathrm{a}}$ rate ratio $(95 \% \mathrm{CI})$ & 0.8 & $(0.6$ to 1.1$)$ & $p=0.1$ & \\
\hline Incidence of CID (diary data) & $81 \%$ & & $89 \%$ & \\
\hline Adjusted $^{\mathrm{a}}$ relative risk $(95 \% \mathrm{CI})$ & 0.9 & $(0.8$ to 1.0$)$ & $p=0.06$ & \\
\hline \multicolumn{5}{|l|}{ Severity of diarrhoea (clinic data) } \\
\hline \multicolumn{5}{|l|}{ Maximum grade adverse event } \\
\hline None & 64 & $(62.7)$ & 46 & $(48.9)$ \\
\hline 1 & 23 & $(22.5)$ & 24 & $(25.5)$ \\
\hline 2 & 9 & $(8.8)$ & 13 & $(13.8)$ \\
\hline 3 & 5 & $(4.9)$ & 10 & $(10.6)$ \\
\hline 4 & 1 & $(1.0)$ & 1 & $(1.1)$ \\
\hline Proportion with grade 1 or higher $\mathrm{AE}$ & $37.3 \%$ & & $50.5 \%$ & \\
\hline Adjusted $^{\mathrm{a}}$ relative risk & 0.7 & ( 0.50 to 0.93$)$ & $p=0.02$ & \\
\hline Proportion with grade 3 or higher AE & $5.9 \%$ & & $11.7 \%$ & \\
\hline Adjusted $^{\mathrm{a}}$ relative risk & 0.5 & $(0.2$ to 1.2$)$ & $p=0.1$ & \\
\hline \multicolumn{5}{|l|}{ Use of anti-diarrhoeal medication (diary) } \\
\hline Yes & $37 / 97$ & $(38.1)$ & $29 / 91$ & $(31.9)$ \\
\hline \multicolumn{5}{|l|}{ Use of anti-diarrhoeal medication (clinic) } \\
\hline Yes & $39 / 102$ & $(38.2)$ & $33 / 94$ & $(35.1)$ \\
\hline
\end{tabular}

$C I D$ chemotherapy-induced diarrhoea, $C I$ confidence interval

${ }^{a}$ Analyses were adjusted for the randomisation stratification factors chemotherapy cycle length and planned chemotherapy regimen
Compliance with study product was recorded in the patient diaries. The proportion of those with diary data available who reported eating $90 \%$ or more of the protocol-required study product was $75 \%$ on both ReCharge and placebo.

\section{Discussion}

This study is the first investigation of the effects of ironsaturated lactoferrin and anhydrous milk fat in humans undergoing cancer therapy. For the primary outcome measure of days with CID, the study found no evidence of clinical benefit, although the direction of the observed difference was in favour of ReCharge.

Interpretation of the result requires scrutiny of the completeness of the patient diary data. Missing data were an issue for the diaries as evidenced by 24 of the 197 participants not providing any information on stool frequency during the study period. Overall, missing data were evenly balanced between the two arms, but only $71 \%$ of ReCharge patients and $72 \%$ of

Table 3 Comparison of quality of life (FACIT-D subscales and total score) in ReCharge and placebo groups

\begin{tabular}{|c|c|c|c|c|c|}
\hline \multirow[t]{2}{*}{ Quality of life scale } & \multicolumn{2}{|c|}{ Adjusted $^{\mathrm{a}}$ means over follow-up } & \multirow[t]{2}{*}{ Adjusted $^{\mathrm{a}}$ difference in means } & \multirow[t]{2}{*}{$95 \%$ confidence interval } & \multirow[t]{2}{*}{$p$ value } \\
\hline & ReCharge & Placebo & & & \\
\hline Physical well-being & 22.49 & 22.25 & 0.24 & $(-0.77$ to 1.25$)$ & 0.64 \\
\hline Social well-being & 23.79 & 23.9 & -0.11 & $(-1.00$ to 0.79$)$ & 0.81 \\
\hline Emotional well-being & 19.26 & 19.73 & -0.48 & $(-1.17$ to 0.22$)$ & 0.18 \\
\hline Functional well-being & 19.01 & 19.82 & -0.81 & $(-1.98$ to 0.36$)$ & 0.17 \\
\hline Diarrhoea subscale & 40.27 & 40.07 & 0.2 & $(-0.94$ to 1.35$)$ & 0.73 \\
\hline FACIT-D total score & 124.8 & 125.5 & -0.7 & $(-4.2$ to 2.7$)$ & 0.67 \\
\hline
\end{tabular}

${ }^{a}$ Analyses were adjusted for the randomisation stratification factors chemotherapy cycle length and planned chemotherapy regimen 


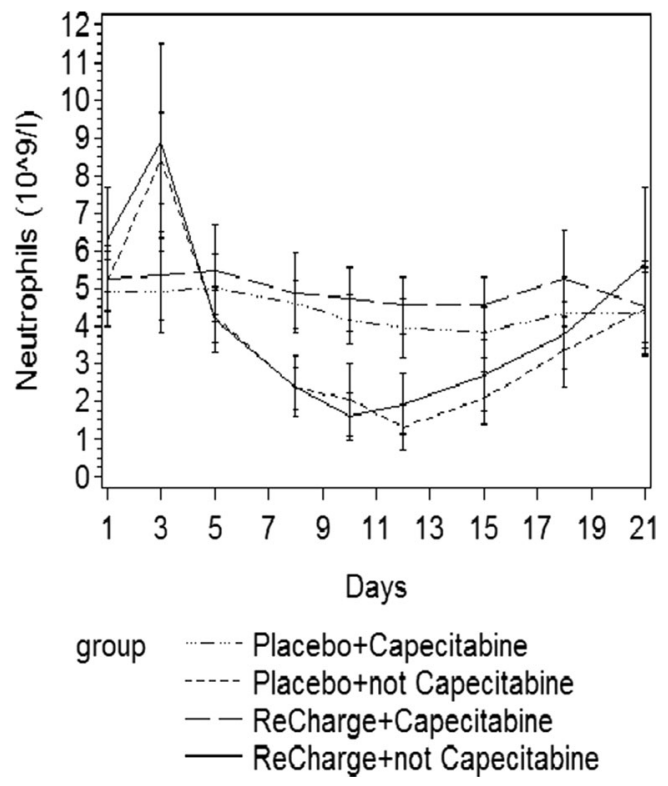

Fig. 2 Mean neutrophil counts and $95 \%$ confidence intervals at each time point by treatment group according to whether or not patients received capecitabine alone (cycle length is 3 weeks only as no patients on 2 -week cycles had capecitabine alone)

placebo patients recorded at least $80 \%$ of the required stool frequency data. The commonest reasons for missing data were the patient being too unwell or forgetting. The majority of the missing data were from patients who had stopped taking chemotherapy and, therefore, technically, were no longer at risk of CID. However, the proportions stopping chemotherapy differed between the treatment and control groups, and there could be some carryover of the effects of chemotherapy, making it difficult to gauge the degree of bias from the missing data. Other cancer treatment studies using daily diaries have reported issues with compliance, particularly for patients with advanced cancer or declining health and compliance is generally better with instruments requiring less frequent completion [18]. A daily diary was chosen for the primary outcome measure to allow better detection of acute, short-term changes compared to instruments using less frequent assessments [18] to better capture the overall impact of diarrhoea, but the degree of missing data has limited the usefulness of this measure.

In contrast, the secondary measure of CID based on adverse events reported in the clinic demonstrated a statistically significant $30 \%$ reduction in CID with ReCharge $(p=0.019)$. The definition of diarrhoea used for the diary and clinic measures was the same (CTCAE version 3 definition of grade 1 diarrhoea is an increase of 1-3 stools per day over baseline frequency). The proportions of CID reported in the clinic were lower than for the diary data (37.3 and $50.3 \%$ on ReCharge and placebo for clinic data and 81 and $89 \%$ for the diary data). This was expected, and indeed one of the reasons for the choice of the diary data for the primary endpoint was the belief that the mild to moderate diarrhoea (that was hypothesised to be prevented by ReCharge) would be underreported in clinic data. In hindsight, the high proportion of patients having CID according to the diary data ( 81 and $89 \%$ ) suggests that the diary measure was too sensitive and perhaps a greater treatment effect might have been observed if a higher threshold was used. However, there are potential biases in the clinic data as well. This information relies on the patient's recollection

Table 4 Numbers of patients experiencing each type of adverse event (as reported at clinic visits and coded according to CTCAE)

\begin{tabular}{|c|c|c|c|c|c|c|c|c|}
\hline & \multicolumn{4}{|c|}{ Any grade } & \multicolumn{4}{|c|}{ Grade 3 or higher } \\
\hline & \multicolumn{2}{|c|}{ ReCharge $(N=102)$} & \multicolumn{2}{|c|}{ Placebo $(N=94)$} & \multicolumn{2}{|c|}{ ReCharge $(N=102)$} & \multicolumn{2}{|c|}{ Placebo $(N=94)$} \\
\hline & $N$ & $\%$ & $N$ & $\%$ & $N$ & $\%$ & $N$ & $\%$ \\
\hline Neutrophils & 12 & $(11.8)$ & 19 & $(20.2)$ & 12 & $(11.8)$ & 18 & $(19.1)$ \\
\hline Platelets & 2 & $(2.0)$ & 5 & $(5.3)$ & 2 & $(2.0)$ & 2 & $(2.1)$ \\
\hline Gastrointestinal (any) & 88 & $(86.3)$ & 80 & $(85.1)$ & 13 & $(12.7)$ & 18 & $(19.1)$ \\
\hline Nausea & 51 & $(50.0)$ & 48 & $(51.1)$ & 1 & $(1.0)$ & 2 & $(2.1)$ \\
\hline Diarrhoea & 38 & $(37.3)$ & 48 & $(51.1)$ & 6 & $(5.9)$ & 11 & $(11.7)$ \\
\hline Vomiting & 25 & $(24.5)$ & 26 & $(27.7)$ & 1 & $(1.0)$ & 3 & $(3.2)$ \\
\hline Constipation & 21 & $(20.6)$ & 23 & $(24.5)$ & 1 & $(1.0)$ & 0 & $(0.0)$ \\
\hline Mucositis (clinical exam) & 6 & $(5.9)$ & 11 & $(11.7)$ & 0 & $(0.0)$ & 3 & $(3.2)$ \\
\hline Mucositis (functional/symptomatic) & 16 & $(15.7)$ & 19 & $(20.2)$ & 1 & $(1.0)$ & 1 & $(1.1)$ \\
\hline Anorexia & 14 & $(13.7)$ & 17 & $(18.1)$ & 0 & $(0.0)$ & 2 & $(2.2)$ \\
\hline Distension & 3 & $(2.9)$ & 2 & $(2.1)$ & 0 & $(0.0)$ & 0 & $(0.0)$ \\
\hline GI other & 4 & $(3.9)$ & 5 & $(5.3)$ & 1 & $(1.0)$ & 0 & $(0.0)$ \\
\hline Any GI or blood adverse event & 88 & $(86.3)$ & 83 & $(88.3)$ & 26 & $(25.5)$ & 34 & $(36.2)$ \\
\hline
\end{tabular}

Comparisons of proportions for each adverse event all gave $p>0.05$ 
over 2 or 3 weeks, and this is likely to be less reliable than the daily diary. However, since the study was blinded to patients and clinical staff, it is likely that recall was equally poor in both treatment groups.

There were no differences between the treatment arms in overall health-related quality of life or in diarrhoea-related quality of life. There was also no statistically significant difference in neutropenia as measured by the area under the curve of neutrophils over time during the first cycle of chemotherapy. However, inspection of the plots of the means (Fig. 2) indicated that the impact of chemotherapy on neutrophil count was restricted to those patients on agents other than capecitabine. In addition, while not statistically significant, the number of neutrophil-related adverse events was lower in the ReCharge group than in the placebo group. Given the relatively small size in this study, we cannot rule out an impact of ReCharge on chemotherapy-induced myelosuppression, so this may be worth further investigation.

Comparisons of treatments for chemotherapy side effects are complicated by withdrawal from chemotherapy. As anticipated, not all patients who entered the study completed their full chemotherapy programme, and furthermore, there was an imbalance in the two arms with fewer ReCharge patients receiving all of their anticipated chemotherapy. It is not possible to conclude whether this lower chemotherapy completion with ReCharge was due to diminished tolerability for chemotherapy in this arm or to other factors, although there was no excess of adverse events for those receiving ReCharge. But, the patterns in withdrawals from chemotherapy may have led to an overestimate of the benefits of ReCharge.

A factor which may have led to an underestimate of the benefit of ReCharge was the need for priming of the gut with study product before the start of chemotherapy. A previous animal study relating to the anti-cancer effects of ironsaturated lactoferrin in C57BL/6 mice reported that administration for at least 2 weeks prior to chemotherapy was necessary to achieve a biological response [5]. The optimal prechemotherapy period of administration in humans is unknown, but in a study in patients, it is necessary to balance the benefit of a longer priming period against an undesirable delay in starting chemotherapy. Initially, the priming period was set at 14 days, but even this led to difficulties in recruitment so it was subsequently changed to $14 \pm 4$ days. It is certainly possible that this period may have been insufficient to optimise the effect of ReCharge. If future studies of ReCharge in the cancer treatment setting are considered, the issue of a lead-in time for ReCharge will be a key consideration.

Measurement of diarrhoea burden and diarrhoea-related quality of life was a challenge for this study and is an area that requires further research. At the time of inception, there were no validated measures of mild to moderate diarrhoea, and while anecdotally recognised as a serious problem by oncologists, there is very little information about the nature and frequency of CID of all grades. Our belief when designing this trial was that the extent of CID was greater than would be reported in the clinic during a standard therapeutic trial and that even low-grade diarrhoea could negatively impact on QoL. The reporting issue was borne out by the difference in prevalence estimates from the diary and clinic data (over $80 \%$ in the diary data and less than $50 \%$ in the clinic data). The substantial literature on measuring quality of life stresses the importance of patient-reported outcomes, but mechanisms to deal with the frequent non-response, particularly in patients who are the most unwell, are under-researched. Literature comparing paper to electronic diaries [19] suggests that both are subject to problems with missing data but that electronic diaries may be more reliably filled out each day. A further challenge is how the analysis should handle patients who withdraw from chemotherapy. A standard intention to treat analysis requires that all patients randomised are included in the analysis. However, patients who have withdrawn from chemotherapy may well have less diarrhoea, so if a product such as ReCharge could enable more patients to stay on chemotherapy because of reduced diarrhoea, the standard ITT analysis may not detect a benefit. Further methodological research is needed to support trials of treatments to reduce the side effects of chemotherapy.

In conclusion, this study did not detect a benefit from ReCharge with the primary efficacy measure of patient diary reported days with CID, but there were methodological issues which could have influenced this outcome. In particular, the adequacy of the patient daily diary record can be called into question as can the degree of patient compliance in taking ReCharge. It should be noted, however, that some of the secondary objectives did show a potential benefit for ReCharge. The incidence of diarrhoea as recorded by the doctor was lower in the ReCharge group, and there was also a suggestion of less neutropenia and fewer adverse effects from neutropenia. However, these results must be interpreted with caution due to the multiple secondary outcome measures (and consequent inflation of the chance of a false positive result) and the possible bias in CTCAE assessment of diarrhoea from differing rates of anti-diarrhoeal medication usage. The effect of the 14 \pm 4 -day lead-in time for ReCharge on the outcome of the study is unknown but may have also contributed to the overall negative result.

Acknowledgments We gratefully acknowledge the contributions of Drs. M. Jameson, R. North and R. Isaacs for recruitment of participants for this study.

Funding The study was sponsored by LactoPharma ${ }^{\mathrm{TM}}$ (A joint venture between Fonterra Cooperative Ltd and Auckland UniServices), and the study product ReCharge was developed and produced by LactoPharma $^{\mathrm{TM}}$. 


\section{References}

1. Rubenstein EB, Peterson DE, Schubert M et al (2004) Clinical practice guidelines for the prevention and treatment of cancer therapy-induced oral and gastrointestinal mucositis. Cancer 100: 2026-2046

2. Sonis ST, Elting LS, Keefe D et al (2004) Perspectives on cancer therapy-induced mucosal injury. Cancer 100:1995-2025

3. Gervasio JM (2010) Diarrhea. In: Tisdale JE, Miller DA (eds) Drug Induced Diseases: Prevention, Detection and Management, 2nd edn. American Society of Health-System Pharmacists, Bethesda, pp 738-759

4. Keefe DM, Schubert MM, Elting LS et al (2007) Updated clinical practice guidelines for the prevention and treatment of mucositis. Cancer 109:820-831

5. Kanwar JR, Palmano KP, Sun X et al (2008) 'Iron-saturated' lactoferrin is a potent natural adjuvant for augmenting cancer chemotherapy. Immunol Cell Biol 86:277-288

6. Sun X, Zhang J, Gupta R, Macgibbon AK et al (2011) Dairy milk fat augments paclitaxel therapy to suppress tumour metastasis in mice, and protects against the side-effects of chemotherapy. Clin Exp Metastasis 28:675-688

7. van't Land B, van Beek NM, van den Berg JJ et al (2004) Lactoferrin reduces methotrexate-induced small intestinal damage, possibly through inhibition of GLP-2-mediated epithelial cell proliferation. Dig Dis Sci 49:425-433

8. Paesano R, Torcia F, Berlutti F et al (2006) Oral administration of $\mathrm{Lf}$ increases hemoglobin and total serum iron in pregnant women. Biochem Cell Biol 84:377-380

9. Di Mario F, Aragona G, Dal BN (2006) Bovine lactoferrin for Helicobacter pylori eradication: an open, randomized, multicentre study. Aliment Pharmacol Ther 23:1235-1240
10. Kaito M, Iwasi M, Fujita N et al (2007) Effect of lactoferrin in patients with chronic hepatitis $\mathrm{C}$ : combination therapy with interferon and ribavirin. J Gastroenterol Hepatol 22:1894-1897

11. Manzoni P, Mostert M, Stronati M (2011) Lactoferrin for prevention of neonatal infections. Curr Opin Infect Dis 24:177-182

12. Kozu T, Iinuma G, Ohashi Y et al (2009) Effect of orally administered bovine lactoferrin on the growth of adenomatous colorectal polyps in a randomized, placebo-controlled clinical trial. Cancer Prev Res (Phila) 2:975-983

13. Parikh PM, Vaid A, Advani SH et al (2011) Randomized, doubleblind, placebo-controlled phase II study of single-agent oral talactoferrin in patients with locally advanced or metastatic nonsmall-cell lung cancer that progressed after chemotherapy. J Clin Oncol 29:4129-4136

14. Troost F, Steijns J, Saris J, Brummer R (2001) Gastric digestion of bovine lactoferrin in vivo in adults. J Nutr 131:201-2104

15. Lumley T, Kronmal R, Ma S (2006) Relative risk regression in medical research: models, contrasts, estimators and algorithms. University of Washington Biostatistics Working Paper Series, Working Paper;293

16. Fleming TR, Richardson BA (2004) Some design issues in trials of microbicides for the prevention of HIV infection. J Infect Dis 190: 666-674

17. Findlay MP, Sharples KJ, Thompson PI et al (2008) A phase II study of capecitabine, oxaliplatin and bevacizumab using a 2 weekly schedule in previously untreated patients with advanced, unresectable colorectal cancer. Ann Oncology 19(Suppl 8):373

18. Gower NH, Rudd RM, Ruiz de Elvira MC et al (1995) Assessment of 'quality of life' using a daily diary card in a randomised trial of chemotherapy in small-cell lung cancer. Ann Oncol 6:575-580

19. Dalea O, Hagen KB (2007) Despite technical problems personal digital assistants outperform pen and paper when collecting patient diary data. J Clin Epidemiol 60:8-17 